\title{
Performance Analysis of Business Processes using Process Mining
}

\author{
Omer S. Dawood \\ Prince Sattam bin Abdulaziz University \\ College of Arts and Science-wadi aldwaser - CS department-KSA \\ Sudan University of Science and Technology-Sudan
}

\begin{abstract}
This paper is aimed to investigate the real time Business process Performance and talent to reinforce the performance. An easy framework was developed to indicate the various steps of analysing and enhancing the process performance. Projected during this paper are a BAM style framework for the real-time business performance management associated an implementation of BAM system model to indicate the pertinence of proposed framework. The goals of business activity observance are to produce real time data concerning the standing and results of varied operations, processes, and transactions. The framework consists from three stages and its facilitates the enhancements of business process.
\end{abstract}

\section{Keywords}

Process mining, BAM, Real Time Performance, Monitoring, Bizagi.

\section{INTRODUCTION}

There are several information systems within the organization like ERP and bpm. It's vital to measure and monitor the performance of these systems instantly to confirm from top quality of the performance and no any delay particularly for giant systems that has huge information. Business activity monitoring (BAM) is software package that aids in observation of business activities, as those activities are enforced in computer systems. The corporation can manage, monitor their business processes, observe the failures or exceptions, and address them in real-time, all these can be handled trough BAM. additionally, since BAM tracks method executions and is aware of after they succeed or fail, it builds up valuable records of behaviour which will result in overall process improvement, whereas conjointly providing a useful tool to manage compliance, assure business transactions, and scale back risk [7].

The term was originally coined by analysts at Gartner, Inc [1]. And refers to the aggregation, analysis, and presentation of real-time data concerning activities within organizations and involving customers and partners. A business activity will either be a business process that's orchestrated by business process management (BPM) software system, or a business process that's a series of activities spanning multiple systems and applications. BAM is associate enterprise resolution primarily meant to supply a time period outline of business activities to operations managers and higher management.

\section{Key Performance Indicator (KPI)}

Key driving factor that implements the strategic objectives of enterprises and it's an analysis index of the core events, it formulates round the enterprise methods, and it's a style of expression for the quantified strategy [4]. Key performance Indicators are accustomed measure staffs' work performance indexes, thus it's the necessary a part of the performance plans.

\section{Business Activity Monitoring (BAM)}

It's provide real-time access to critical business performance indicators to enhance the speed and effectiveness of business operations [1][8]. The BAM aimed to supply real-time info regarding the standing and results of varied business operations, processes, and transactions. BAM cut back the delays, bottlenecks and inefficient use of labour and materials, whereas providing real-time monetary and performance knowledge [2][10]. BAM permits a business to watch its business processes, and connected business events being generated in real-time, and provides an assessment of business process supported pre-defined KPIs [9]. this enables bigger operational visibility of the business to relevant process owners for assessment and decision-making via time period vital metrics with relevancy frequencies of such errors and their potential harm on process performance, and alternative dimensions like value, schedules, and so on .[5] [6]. A key construct of BAM is that the aggregation of basic Events regarding this state and therefore the results of business processes into quantitative measures, alleged Key Performance Indicators (KPIs).BAM is visually displays the foremost vital info required to attain one or additional objectives, consolidated and organized on one screen so the data is monitored at a look [3].

\section{Case Study}

A small indebtedness bpm system has been developed and measured victimisation Bizagi BPMS The system has four pool and three phases, the reception receives invoice and send it to financial help that match the invoice with purchase order, if match then check if the product or service is approved or not, if approved send the invoice to the accounting to update ERP. If invoice not matched with purchase order or if the product or service isn't approved come back the invoice to the provider. The process| of approved return the invoice to the provider is sub process that consists from three processes, initial process is justify the rejection process, if the invoice is rejected, the one that executes this task should indicate, the one that executes

This task should indicate rejection reasons for rejection either as a result of the data doesn't match the purchase order or as a result of the product or Services are unsatisfactory. Second process is Inform provider this can be done mechanically by sending associate email to the provider with the rationale for the rejection of the invoice and therefore the comeback of product, if the product haven't to be came back then finish the task, else move to come back products and to the products are came back to the supplier's address. 


\section{RELATED WORK}

Process mining refers to the analysis of the process log or data admire the process of a business and extracting the specified info [14]. Process mining technology consists of 3 main types - discovery, correspondence, and improvement [15]. That is, process mining isn't solely capable of discovering process models however additionally of characteristic deviations between models and log, and of conducting performance analyses [16]. Process mining permits for the extraction of necessary info associated with the process and permits one to derive, monitor, and improve the particular method [17]. As a result of process mining technology analyses the $\log$ that is already within the variety of data, it will save time and cut back the prices of data assortment. Additionally, method mining technology solely analyses recorded data that prevents distortion of data and ensures accuracy and objectivity [18].

Business activity monitoring (BAM) was first outlined by Gartner because the idea of providing real-time access to crucial business performance indicators to enhance the speed and effectiveness of business operations [11]. The goal of BAM is to produce real-time data regarding the status and results of assorted business operations, processes, and transactions [12]. Enterprise-wide task of BAM is to scale back or eliminate delays, bottlenecks and inefficient use of labour and materials, whereas providing real-time financial and performance information [13]. Representative feature of BAM is that it monitors several enterprise systems at the same time and displays exceptional scenario on the dashboard if symptoms of drawback are known by pre-defined rules.

Several approaches are planned within the literature for the discovery of declarative process models. In Ref. [19] the authors present an approach that permits the user to pick out from a group of predefined Declare templates those to be used for the discovery. Maggi et al. propose an evolution of this approach in ref. [20] to enhance performance by preprocessing the event $\log$ with frequent pattern mining techniques. Different approaches to enhance the performance of process mining are given in Refs. [21]. in addition, there are post-processing approaches that aim at simplifying the ensuing Declare models in terms of redundancy elimination [22] and illumination [23]. The approach proposed in ref. [24] permits for the specification of rules that transcend the normal Declare templates. In Ref. [25], an approach for analysing event $\operatorname{logs}$ with regular Declare, an extension of Declare that depends on regular automata, is delineate. The add ref. [26] initial coated the info perspective in declarative process mining, though this approach solely permits for the discovery of discriminative activation conditions. In essence, the main focus of the said approaches is control-flow with extensions to hide knowledge while not analysing resource-related data.

\section{METHODOLOGY}

The Research deeply takes the process of monitoring the performance of business process. A simple case study was developed using Bizagi business process management tool, and the performance was monitored and enhanced according to the developed framework. The Framework consists from three components as follow:

\subsection{Designing Business Process}

In this stage the intended business process that satisfy the organization requirements and follow the needed rules will be designed. There are many tools and techniques that used to design business process that needed in the organization. In our case the Bizagi Process modeller used to design the process. Sometimes we need to enhance the business process to obtain better performance.

\subsection{Analysis of Process Performance}

There are three sub-process under these module, firstly the KPI of each process is defined according to the performance plan, secondly the achieved performance will be measured according to the KPI defined in previous stage, this can help as indicator of maturity of business process and if its satisfy the needed performance, and finally the dashboard that shows the performance was displayed, in the dashboard the result of performance measurements is clearly shown, then the business analysts or manager can make a decision either this performance is a good or need some enhancement. 


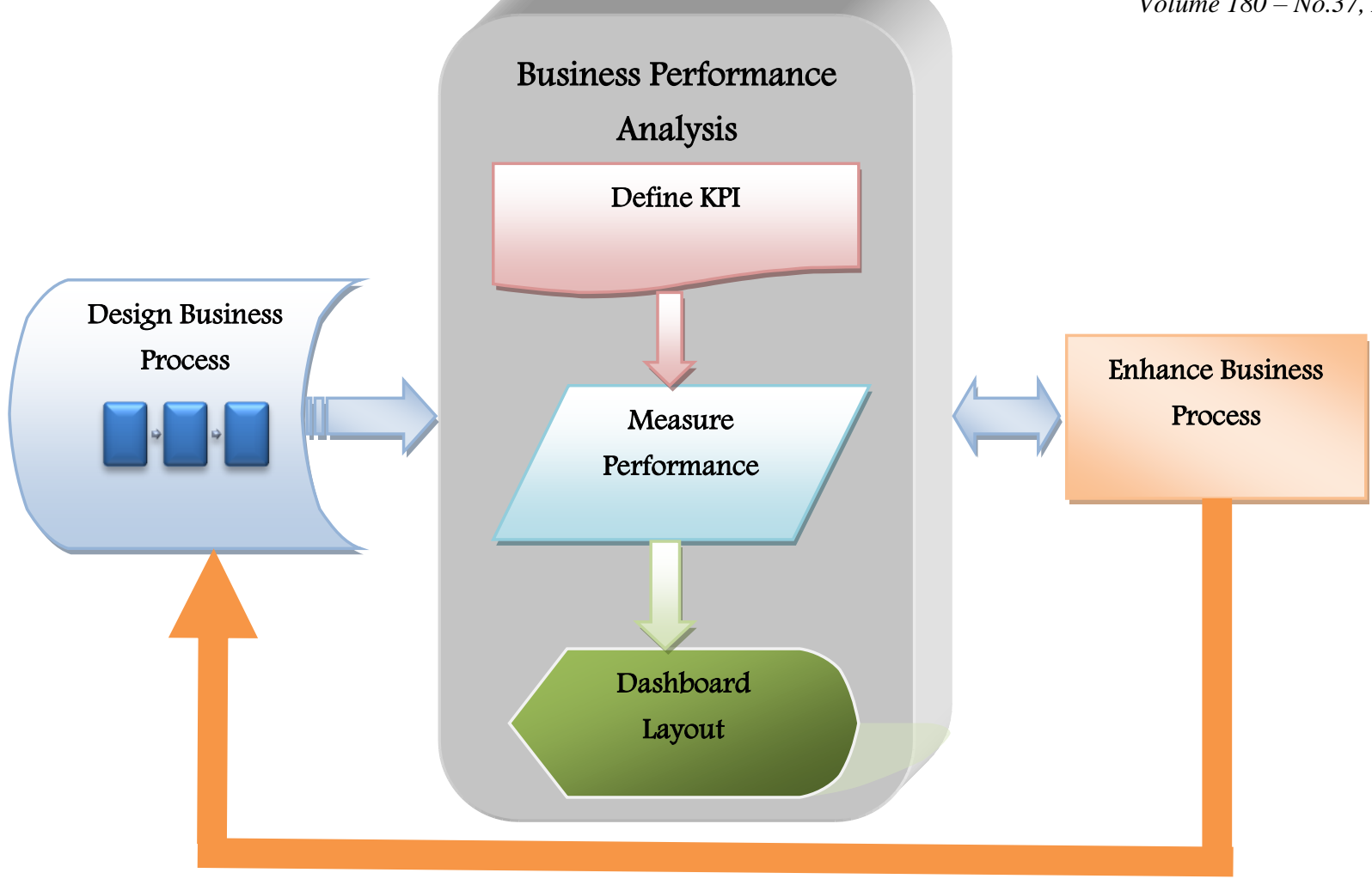

Figure 1: Framework

\subsection{Enhance the performance}

This is last stage of our model, based on the dashboard layout some enhancement may be needed, there are two ways to enhance performance, through re-enhance KPI or re-enhance the Business process.

Applying this frame work shows how we can make a real time analysis of business process using BAM, and how we can improve or enhance the process performance. This framework is applied by using bizagi tools and its shows a good enhancement in business process

\section{CONCLUSION AND FUTURE WORK}

In this paper I proposed a framework that performs monitoring of business activity monitoring (BAM) of business processes running on prime of a Service-Oriented design. Besides providing up-to-date dashboard data regarding this process performance, the most goal of our framework is to enable what we consult with as dependency analysis, i.e., an analysis of the most factors that influence the business process and create it violate its performance targetsFinally, we commit to investigate choices for mapping the output to graphical process modelling notations to extend readability.

\section{REFERENCES}

[1] Stefanie e at "Business Process Management: 9TH INTERNATIONAL CONFERENCE," Chennai . India, eISSN 1611-3349, April 2011. (references)

[2] Jin Gu Kang and Kwan Hee Han, "A Business Activity Monitoring System Supporting Real-Time Business Performance Management", [Third 2008 International Conference on Convergence and Hybrid Information Technology korea, Vol 1, 2008].

[3] Jan-Philipp e at, "Extending BPMN for Business Activity Monitoring" [2012 45th Hawaii International Conference on System Sciences, 2012].
[4] Wang Pan and He Wei, "Research on key performance indicator (KPI) of business process", [2012 Second International Conference on Business Computing and Global Informatization, Shanghai, 2012].

[5] W.Schmidt,Business Intelligence and Performance Management,Springer-Verlag London 2013.

[6] http://www.redbooks.ibm.com/redbooks/pdfs/sg247638. pdf, July 2008

[7] James Crump, business activity monitoring (bam): The New Face of BPM, June 2006.

[8] BusinessActivityMonitoring.http://www.fujitsu.com/dow nloads/SVC/fc/fs/bam.pdf.Access date:[Second of Nov, 2015-6:10 PM]

[9] Peter Rausch - Alaa F. Sheta - Aladdin Ayesh, Business Intelligence and Performance Management, 2013.

[10] Kapil Pant, Matjaz B. Juric,Business Process Driven SOA using BPMN and BPEL,August 2008.

[11] [11] McCoy, D., Schulte, R., Buytendijk, F.,Rayner, N., and Tiedrich, A., "Business Activity Monitoring: The Promise and Reality", Gartner, Gartner's Marketing Knowledge and Technology Commentary COM-139992, 2001

[12] [12] Wikipedia, "Business Activity Monitoring", http://en.wikipedia.org/wiki/Business_activity_monitorin g, 2017, access time:[10/3/2017, 7:30 pm].

[13] [13] Govekar, M. and Schulte, R., "BAM Architecture: More Building Blocks Than You Think", Gartner, AV15-5070, 2002.

[14] R.P. Bose, W.M.P. van der Aalst, Dealing with concept drifts in process mining, Neural Netw. Learn. Syst. IEEE Trans. 25 (2014) 154-171. 
[15] R. Mans, W.M.P. van der Aalst, R. Vanwersch, Process Mining in Healthcare: Evaluating and Exploiting Operational Healthcare Processes, Springer International Publishing, 2015, pp. 17-26.

[16] M. Cho, M. Song, S. Yoo, A systematic methodology for outpatient process analysis based on process mining, Int. J. Ind. Eng. 22 (2015) 480-493.

[17] W.M.P. van der Aalst, M.H. Schonenberg, M. Song, Time prediction based on process mining, Inf. Syst. 36 (2011) 450-475.

[18] M. Song, W.M.P. van der Aalst, Towards comprehensive support for organizaional mining, Decis. Support Syst. 46 (2008) 300-317.

[19] F.M. Maggi, A. Mooij, W. van der Aalst, User-guided discovery of declarative process models, IEEE Symposium on Computational Intelligence and Data Mining, 2011. pp. 192-199. http://dx.doi.org/10.1109/CIDM.2011.5949297.

[20] F.M. Maggi, J.C. Bose, W. van der Aalst, Efficient discovery of understandable declarative process models from event logs, Int. Conf. on Advanced Information 89 (2016) 87-97 97 Systems Engineering (CAiSE), 7328, 2012. pp. 270-285. http://dx.doi.org/10. 1007/978-3-64231095-9_18.

[21] M. Westergaard, C. Stahl, H. Reijers, UnconstrainedMiner: Efficient Discovery of Generalized
Declarative Process Models, Eindhoven University of Technology. 2013, URL https://publications.hse.ru/en/preprints/117624631.

[22] J.C. Bose, F.M. Maggi, W. van der Aalst, Enhancing declare maps based on event correlations, Int. Conf. on Business Process Management (BPM), 8094, 2013. pp. 97-112. http://dx.doi.org/10.1007/978-3-642-40176-3_9.

[23] F.M. Maggi, J.C. Bose, W.M. van der Aalst, A knowledge-based integrated approach for discovering and repairing declare maps, Int. Conf. on Advanced Information Systems Engineering (CAiSE), 7908, 2013 pp. 433-448. http://dx. doi.org/10.1007/978-3-64238709-8_28.

[24] F. Chesani, E. Lamma, P. Mello, M. Montali, F. Riguzzi, S. Storari, Exploiting inductive logic programming techniques for declarative process mining, Trans. Petri Nets and Other Models of Concurrency 2 (2009) 278 295. http://dx.doi. org/10.1007/978-3-642-00899-3_16.

[25] F.M. Maggi, Discovering metric temporal business constraints from event logs, Int. Conf. on Perspectives in Business Informatics Research (BIR) 194, Springer. 2014, pp. 261-275. http://dx.doi.org/10.1007/978-3-31911370-8_19.

[26] F.M. Maggi, M. Dumas, Discovering data-aware declarative process models from event logs, Int. Conf. on Business Process Management (BPM), 8094, 2013. pp. 1-16. http://dx.doi.org/10.1007/978-3-642-40176-3_8. 